ARTICLE

\title{
Efficient rational modification of non-ribosomal peptides by adenylation domain substitution
}

\author{
Mark J. Calcott (10) 1,2, Jeremy G. Owen ${ }^{1,2} \&$ David F. Ackerley (iD ${ }^{1,2 凶}$
}

Non-ribosomal peptide synthetase (NRPS) enzymes form modular assembly-lines, wherein each module governs the incorporation of a specific monomer into a short peptide product. Modules are comprised of one or more key domains, including adenylation ( $A$ ) domains, which recognise and activate the monomer substrate; condensation (C) domains, which catalyse amide bond formation; and thiolation $(T)$ domains, which shuttle reaction intermediates between catalytic domains. This arrangement offers prospects for rational peptide modification via substitution of substrate-specifying domains. For over 20 years, it has been considered that $\mathrm{C}$ domains play key roles in proof-reading the substrate; a presumption that has greatly complicated rational NRPS redesign. Here we present evidence from both directed and natural evolution studies that any substrate-specifying role for $\mathrm{C}$ domains is likely to be the exception rather than the rule, and that novel non-ribosomal peptides can be generated by substitution of $A$ domains alone. We identify permissive $A$ domain recombination boundaries and show that these allow us to efficiently generate modified pyoverdine peptides at high yields. We further demonstrate the transferability of our approach in the PheATEProCAT model system originally used to infer $\mathrm{C}$ domain substrate specificity, generating modified dipeptide products at yields that are inconsistent with the prevailing dogma.

\footnotetext{
${ }^{1}$ School of Biological Sciences, Victoria University of Wellington, Wellington, New Zealand. ${ }^{2}$ Centre for Biodiscovery and Maurice Wilkins Centre for Molecular Biodiscovery, Victoria University of Wellington, Wellington, New Zealand. ${ }^{凶}$ email: david.ackerley@vuw.ac.nz
} 
T he earliest reported attempts to create artificial nonribosomal peptide synthetase (NRPS) enzymes were substitutions of A-T domains into the second and seventh modules of the NRPSs involved in the biosynthesis of the lipopeptide surfactin ${ }^{1,2}$. Although modified lipopeptides were detected using mass spectrometry, in each case the yield of modified lipopeptide was substantially diminished, to only trace levels ${ }^{2}$. Evidence that $\mathrm{C}$ domains exhibit stringent specificity toward the acceptor substrate activated by their cognate (downstream) A domains offered an explanation for the reduced yields ${ }^{3,4}$. This evidence was based on NRPS enzymes artificially loaded with amino-acyl CoA or aminoacyl-N-acetylcysteamine thioesters, which mimic an amino acid attached to a $\mathrm{T}$ domain. Soon after, a substrate binding pocket of the $\mathrm{C}$ domain was suggested to play a role in controlling the direction of biosynthesis ${ }^{5}$. Subsequent efforts at substituting cognate $\mathrm{C}$-A domains together enjoyed modest success, reinforcing the belief that this was necessary to bypass $\mathrm{C}$ domain substrate specificity ${ }^{6,7}$. Since then the most successful engineering attempts have focused on substituting $\mathrm{C}-\mathrm{A}$ domains together ${ }^{8,9}$ or A-T-C domains with the condition of not disrupting $\mathrm{C}$ domain acceptor site specificity ${ }^{10-12}$. It is now widely accepted in the field that successful domain substitution requires working within the constraints imposed by C domain specificity ${ }^{8,10,13}$.

Until now, our own work using pyoverdine as a model system has been consistent with this dogma. Pyoverdine from Pseudomonas aeruginosa PAO1 is a UV-fluorescent siderophore formed from an 11-membered peptide, encoded by NRPS modules that will here be referred to as Pal-Pall (Fig. 1a, c). We observed that five out of five synonymous A domain substitutions into PvdD (an NRPS comprised of modules Pa10 and Pa11, both of which specify L-Thr as the native substrate $)^{14}$ yielded detectable pyoverdine products, versus zero of nine substitutions of A domains specifying alternative residues $^{9,15}$. In contrast, non-synonymous C-A domain substitutions generated modified pyoverdines in three out of ten cases, suggesting that a $\mathrm{C}$ domain with compatible acceptor site specificity was required for functionality $9,15,16$.

We now show that our previous failure to generate modified pyoverdines by A domain substitution is surmountable by use of more effective recombination boundaries, and that the $\mathrm{C}$ domains in the pyoverdine NRPS system do not impose stringent proofreading constraints. We perform comprehensive evolutionary analyses across three different bacterial genera that are consistent with this conclusion, indicating that non-ribosomal peptide divergence is primarily driven by recombination of $\mathrm{A}$ domains or sub-domains, independent of partner C domains. Tellingly, we show that we can also incorporate leucine as the acceptor residue in the tyrocidine PheATE/ProCAT dimodular NRPS model (Fig. 1b, d), which was previously found not to accept artificiallyloaded leucine thioesters at this position-a key observation underpinning the original hypothesis that $\mathrm{C}$ domains exhibit stringent selectivity for the acceptor substrate 3 .

\section{Results}

Investigating C domain proofreading via semi-rational DNA shuffling. Previous structural biology and bioinformatics approaches to identify residues involved in the presumed substrate specificity of $\mathrm{C}$ domains have been unsuccessful ${ }^{17-19}$. We instead adopted a semi-rational shuffling strategy, by combining regions of DNA encoding $\mathrm{C}$ domains that incorporate different amino acid substrates, then seeking to retrospectively identify important substrate-defining residues. For this we selected the Lys-specific module Pa8 and the Thr-specific module Pa11, as we a

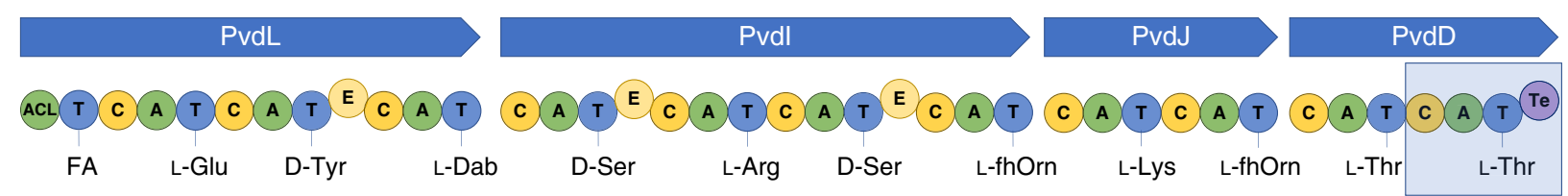

b

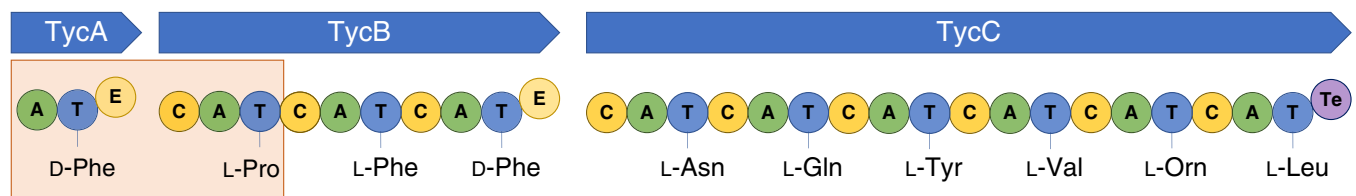

C

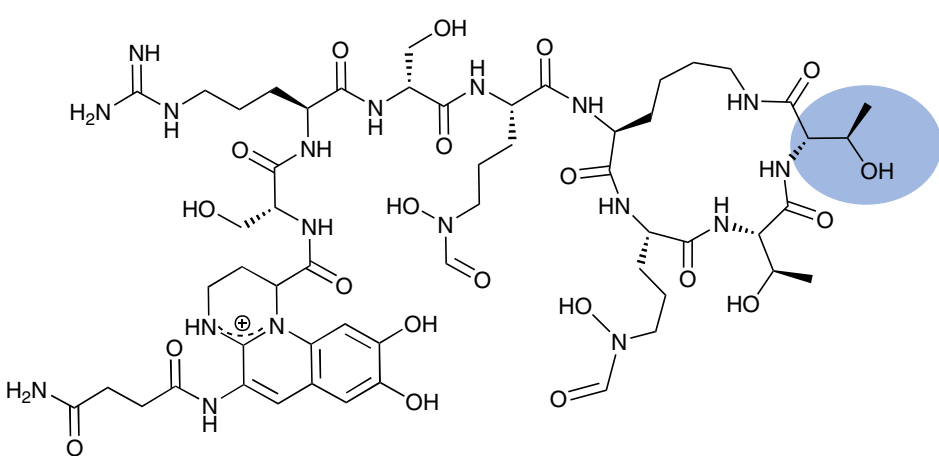

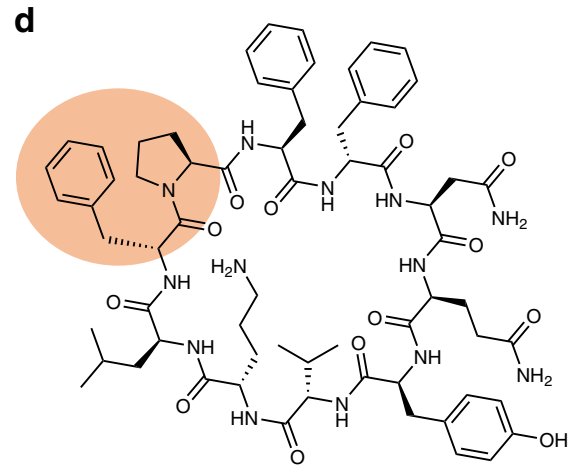

Fig. 1 NRPS assembly lines and products relevant to this work. Depicted are the biosynthetic gene clusters (blue arrows) and their encoded NRPS assembly lines involved in the biosynthesis of $\mathbf{a}$ pyoverdine and $\mathbf{b}$ tyrocidine, and the corresponding chemical structures for $\mathbf{c}$ pyoverdine and $\mathbf{d}$ tyrocidine. The NRPS module Pa11 is highlighted in blue in $\mathbf{a}$, as is the corresponding L-Thr residue that it incorporates into pyoverdine in c. Likewise, the two modules that were extracted from the tyrocidine NRPS system by Belshaw et al. ${ }^{3}$ and used to generate their PheATE/ProCAT model system are highlighted in orange in $\mathbf{b}$, as are the D-Phe and L-Pro residues these incorporate into tyrocidine in $\mathbf{d}$. NRPS domains are labelled as follow: ACL acyl-CoA ligase, C condensation, A adenylation, T thiolation, E epimerisation, and Te thioesterase. The following non-standard amino acid abbreviations are used: Dab, 2,4diaminobutyric acid; fhOrn, $\mathrm{N}^{5}$-formyl- $\mathrm{N}^{5}$-hydroxyornithine; Orn, ornithine. 
had previously found a pyoverdine with Lys at position 11 could be generated by substituting the C-A domains (but not the A domain) from $\mathrm{Pa} 8$ into $\mathrm{Pa} 11$ of $\mathrm{PvdD}^{9}$. At the time, we interpreted this result as showing that the $\mathrm{Pa} 8 \mathrm{~A}$ domain can function within the PvdD environment, but only when paired with a Lysspecifying $\mathrm{C}$ domain. An added attraction of the model is that the $\mathrm{Pa} 8$ and $\mathrm{Pa} 11 \mathrm{C}$ domains appear to be paralogs, as they are nearly identical apart from three stretches of low sequence identity (Supplementary Fig. S1a). We reasoned that these low-identity regions were likely to contain substrate-specifying residues, and a homology model based on the $\mathrm{C}$ domain from $\mathrm{TycC}$ (pdb: $2 \mathrm{JGP}^{20}$ ) suggested the three regions could be shuffled effectively, with only a minimal number of amino acid perturbations introduced (Fig. 2a; Supplementary Fig. S1b, c).

DNA blocks spanning each of the three variable regions of the $\mathrm{Pa} 8$ (presumed K-specific) and Pa11 (presumed T-specific) C domains were shuffled to yield all eight possible combinations (TTT, KTT,..., KKK; Fig. 2b). Each shuffled C domain was then introduced into a $p v d D$ gene construct, immediately upstream of either the native Pa11 or a substituted $\mathrm{Pa} 8 \mathrm{~A}$ domain (Fig. 2b; Supplementary Fig. S2). We observed that region 3 played a dominant role in defining the substrate compatibility of the recombinant $\mathrm{C}$ domains. With the exception of the recombinant $\mathrm{C}$ domain KKT, which was not functional in association with either A domain, $\mathrm{C}$ domains that contained region 3 from $\mathrm{Pa} 11$ were substantially more active in partnership with the Pa11 A domain, and only $\mathrm{C}$ domains that contained region 3 from $\mathrm{Pa} 8$ were active in partnership with the $\mathrm{Pa} 8 \mathrm{~A}$ domain (Fig. 2c; Supplementary Fig. S3). These data suggested that region 3 contains key specificity-determining residues. However, it is important to note that the recombination point between the $\mathrm{C}$ and A domains was near the A1 motif. This meant region 3 of the $\mathrm{C}$ domain was always substituted in association with the corresponding loop that delineates $\mathrm{C}$ and $\mathrm{A}$ domains and is often referred to as a linker region ${ }^{10,21}$. The linker region is an approximately 36 residue sequence that begins at the $\mathrm{C}$-terminus of the final helix of the $\mathrm{C}$ domain, and extends to the first helix of the A domain. When designing this experiment we considered the linker was unlikely to be a significant factor, as there is no structural basis for considering that the linker region could be involved in acceptor site specificity, and the linker region has appeared unimportant in previously successful synonymous substitutions $9,10,15$

Region 3 contains 38 non-identical residues between the $\mathrm{Pa} 8$ and Pa11 C domains (Supplementary Fig. S1a). With the goal of narrowing down the key substrate defining elements, proximal clusters of residues within the $\mathrm{C}$ domain of the Thr-specific module Pall were substituted by the corresponding residues within Pa8 (Fig. 2d; Supplementary Fig. S4). These substitutions focused on clusters of 6 (Shell 1, Fig. 2d) or 12 (Shell 1 and 2, Fig. 2d) residues closest to the catalytic histidine and/or the loop extending across the solvent channel. We also generated a control (Linker, Fig. 2d) in which the Pa11 C domain was fused to the linker region from module $\mathrm{Pa} 8$. Surprisingly, modifications to the $\mathrm{C}$ domain had little effect on pyoverdine production, however changing the Pa11 linker region to that from $\mathrm{Pa} 8$ was sufficient to allow the native PvdD C domain to function efficiently with the $\mathrm{Pa} 8$ lysine-specifying A domain (Fig. 2e; Supplementary Fig. S5). The resulting pyoverdine species was produced in high yield and contained lysine at position 11. Conceptually, substituting the A domain together with its cognate linker is just an A domain substitution that uses a different recombination boundary (i.e. at the very $\mathrm{C}$-terminal end of the $\mathrm{C}$ domain as opposed to the recombination sites within the linker previously used by us ${ }^{9,15}$ and Bozhüyük et al. $^{10}$ ). Identification of a more permissive recombination boundary that did not exchange any plausible proof-reading residues $^{21}$ was inconsistent with the hypothesis that $\mathrm{C}$ domain acceptor site specificity had caused our previous $\mathrm{A}$ domain substitutions to be non-functional ${ }^{9,15}$.

Assessing the efficiency of A domain substitutions. Our previous $\mathrm{C}-\mathrm{A}$ substitutions in pyoverdine module Pa11 had a combined success rate of only $3 / 10$ constructs yielding a detectable pyoverdine product, with two of these being in very low yield $^{9,15,16}$. To test whether A domain substitution using our new upstream recombination boundary was a more efficient strategy, we generated the equivalent linker $+\mathrm{A}$ domain substitution constructs for each of our three previously successful C-A domain substitutions. In each case, the pyoverdine yield was increased by substituting the linker and A domain together (Fig. 3a; Supplementary Figs. S6, S7). We then tested whether we could efficiently produce other modified pyoverdines, by randomly selecting nine A domains that activate substrates other than Thr from Pseudomonas species within the antiSMASH database ${ }^{22}$, and substituting them into module Pa11 (Supplementary Table 1; Supplementary Fig. S8). Six of these A domain substitution variants gave modified pyoverdines at high yields (Fig. 3b; Supplementary Fig. S9). Collectively, these results confirmed not only that substitution of an A domain without the corresponding $\mathrm{C}$ domain is possible, but that it can result in improved success rates and yields compared to C-A domain substitutions.

A domain substitution has driven NRPS diversification in nature. Our success in generating modified pyoverdines via A domain substitution led us to consider whether this approach might be applicable to other pathways. Compared to natural evolution, the number of substitutions that can be created in the lab is infinitesimal, and we reasoned that we could gain valuable insight by investigating whether A domain substitution has occurred frequently during natural NRPS diversification. The tight acceptor site specificity originally proposed by Belshaw et al. $^{3}$ has fuelled speculation that $\mathrm{C}$ and $\mathrm{A}$ domains are likely to

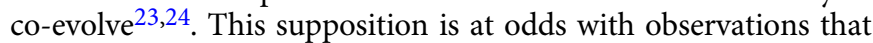
complete or partial A domain substitution has driven diversification of the microcystin ${ }^{25}$, aeruginosin ${ }^{26}$, hormaomycin ${ }^{27}$ and lipo-octapeptide ${ }^{28}$ biosynthetic pathways. The microcystins are a particularly interesting example, as diversification by A domain substitution has been especially prevalent ${ }^{25}$ despite in vitro assays suggesting the $\mathrm{C}$ domain may play an extended gatekeeper role in controlling substrate specificity ${ }^{29}$. We consider it pertinent to note that the latter study also demonstrated that A domains in vitro may adenylate a very different repertoire of amino acid substrates if they are purified in isolation than when they are purified in association with their partner $\mathrm{C}$ domain (a second example of this in vitro inconsistency was reported soon afterward for SulM from the sulfazecin monobactam gene cluster ${ }^{30}$ ). As adenylation is independent of any catalytic role of the $\mathrm{C}$ domain, the inconsistency has been attributed to indirect conformational effects ${ }^{30}$, which in turn suggests that isolated A domains may appear in vitro to be more promiscuous than they actually are in vivo. Thus, while it is possible that diversification via A domain substitution in the microcystin, aeruginosin, hormaomycin and lipo-octapeptide pathways has been enabled by unusually relaxed-specificity $\mathrm{C}$ domains, our experimental success in generating modified pyoverdines led us to consider that these examples might not be so unusual, and that A domain substitution might be a more global phenomenon driving NRPS diversification.

We first investigated this by constructing phylogenetic trees based on the $\mathrm{C}$ and $\mathrm{A}$ domains from NRPS enzymes involved in the biosynthesis of four different pyoverdines (from strains of $P$. 
a

Highlighted regions of the $\mathrm{C}$ domains

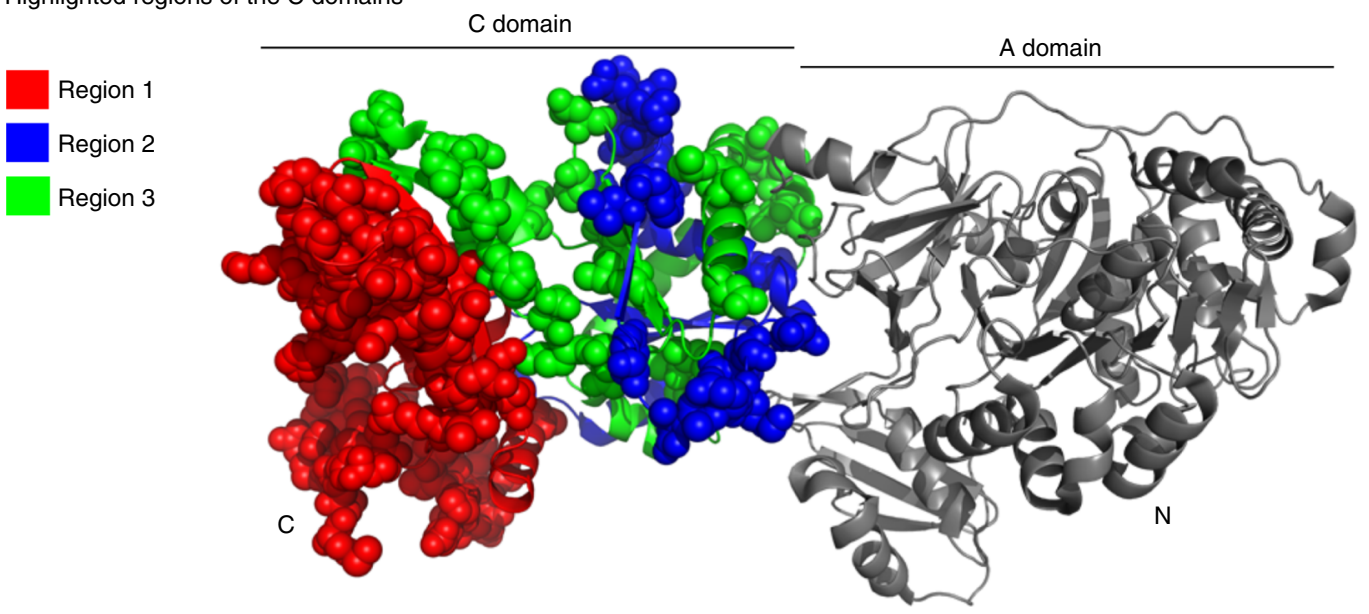

b

Semi-rational shuffling approach
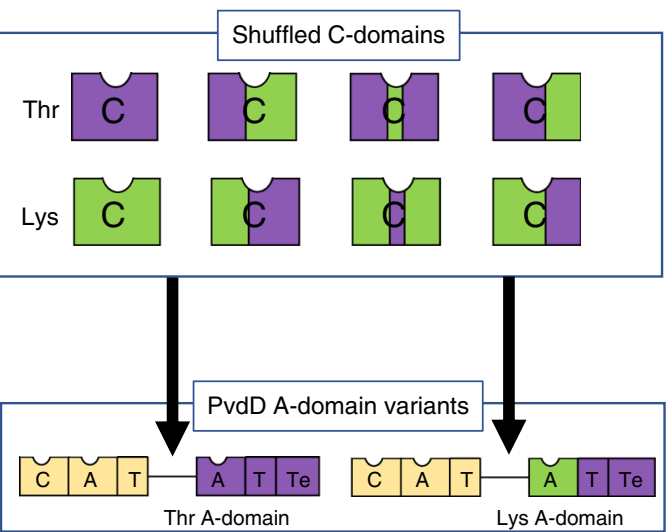

d Modifications to region 3

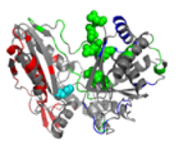

Shell 1

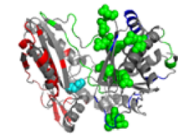

Shell 1 and 2

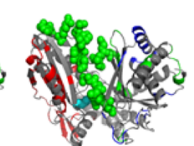

Loop

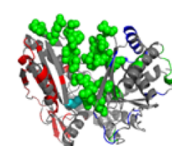

Shell 1 and loop

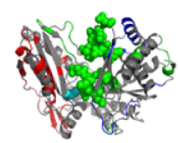

Shell 1 and edges of loop

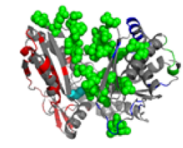

Shell 1, 2 and loop

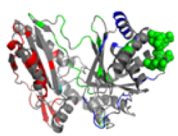

Linker
C

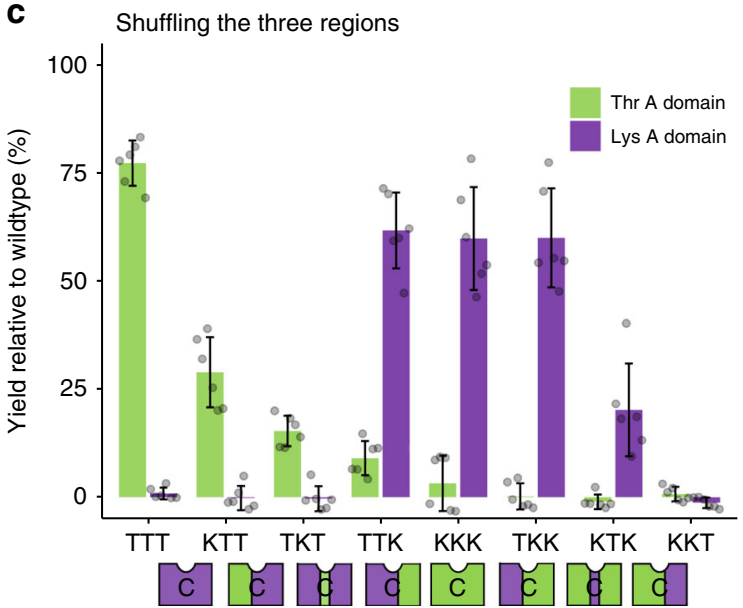

e

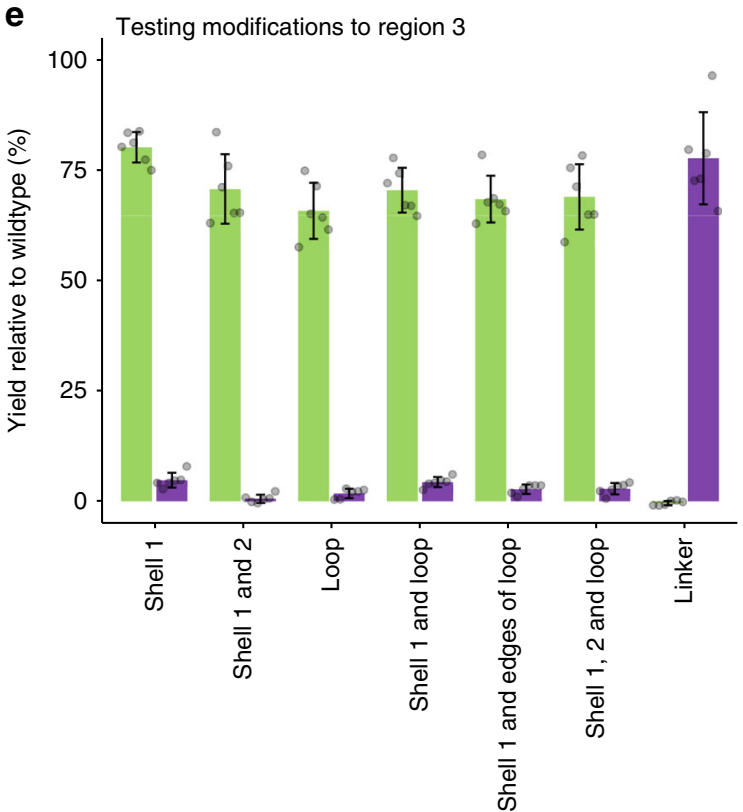

aeruginosa, Pseudomonas putida, Pseudomonas fluorescens and Pseudomonas syringae). This revealed strong clustering of $\mathrm{A}$ domains by substrate specificity, providing evidence that $\mathrm{C}$ and $\mathrm{A}$ domains had evolved independently (Supplementary Fig. S10). To perform a more global analysis of whether $\mathrm{C}$ and A domains have evolved independently, we downloaded the sequences of all
NRPS gene clusters available within the antiSMASH database ${ }^{22}$ for the genera Pseudomonas, Streptomyces and Bacillus. The sequences were extracted, clustered at $95 \%$ identity and aligned to give a total of 437,370 and $213{ }^{\mathrm{L}} \mathrm{C}_{\mathrm{L}}$-A-T tri-domain sequences for Pseudomonas, Bacillus and Streptomyces species, respectively. Analysis with TreeOrderScan ${ }^{31,32}$, which assesses $400 \mathrm{bp}$ 
Fig. 2 DNA shuffling reveals a permissive recombination boundary for A domain substitution. a The three variable regions (coloured red, blue and green in accordance with shading used in Supplementary Fig. S1) of the Pa8 and Pa11 C domains mapped onto the structure of the C-A domains derived from PDB: 2VSQ21. Residues differing between the Pa8 and Pa11 C domains are shown as spheres. $\mathbf{b}$ Overview of the semi-rational shuffling approach used to narrow down substrate specifying regions. The three variable regions of the $C$ domains from modules Pa8 (green) and Pa11 (purple) were shuffled in every combination to create eight variant $C$ domains. Each of these was inserted into a plasmid containing a pvdD gene lacking the Pa11 $C$ domain (left-hand construct), and a plasmid containing a pvdD gene lacking the Pa11 C domain, and in which the Pa11 A domain had additionally been replaced by the Lysspecific A domain from Pa8 (right-hand construct). c Pyoverdine production from pvdD deletion strains transformed by the variant $p v d D$ genes from $\mathbf{b}$ was assessed by measuring absorbance at $400 \mathrm{~nm}$ relative to a wild-type $P$. aeruginosa strain. $\mathbf{d}$ Homology models highlighting (in light green) clusters of residues that were substituted as groups within the third region of Pa11 together with the linker-only substituted control. e Pyoverdine production from pvdD deletion strains transformed by the variant $p v d D$ genes from panel $d$ was assessed by measuring absorbance at $400 \mathrm{~nm}$ relative to a wild-type $P$. aeruginosa strain. For $\mathbf{c}$ and $\mathbf{e}, n=6$ independent experiments and data are presented as mean values \pm SD. Source data are provided as a Source Data file.

a

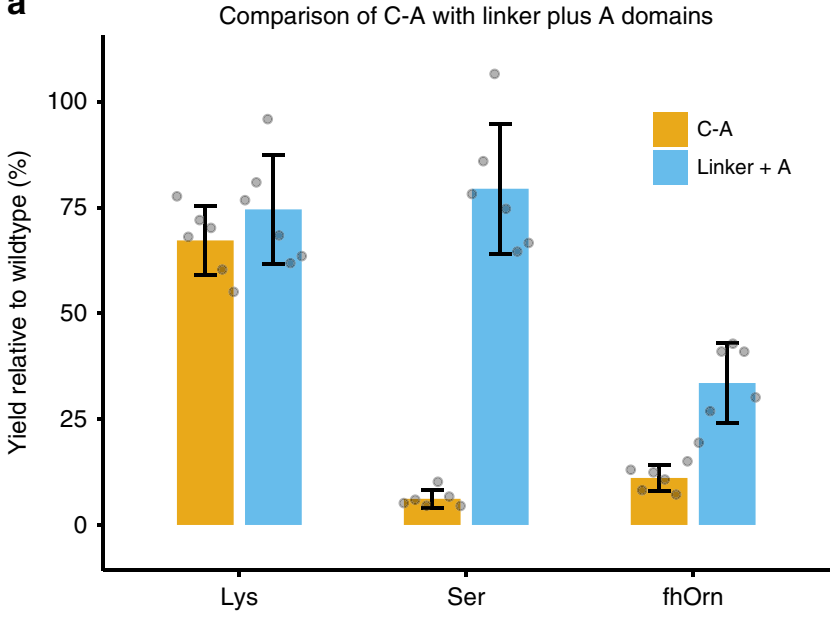

b

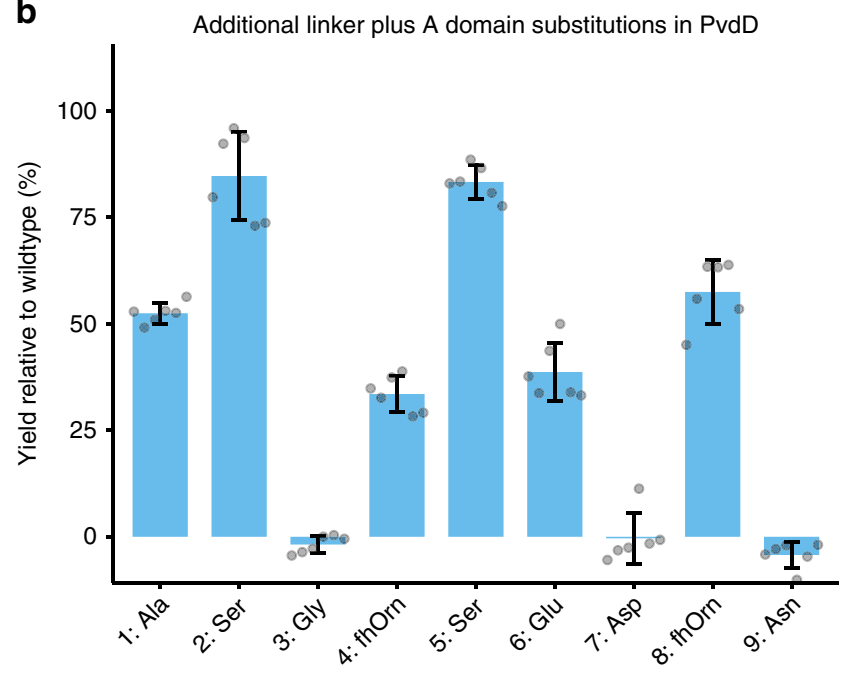

Fig. 3 Efficient generation of modified pyoverdines by linker + A domain substitutions. a Pyoverdine production for C-A domain substitution strains compared with the corresponding linker $+A$ domain substitution strains. b Pyoverdine production for nine additional $A+$ linker domain substitution variants. Pyoverdine production was assessed by measuring absorbance at $400 \mathrm{~nm}$ relative to a wild-type $P$. aeruginosa strain. In all cases, $n=6$ independent experiments and data are presented as mean values \pm SD. Source data are provided as a Source Data file. The abbreviation fhOrn represents $\mathrm{N}^{5}$-formyl-N5-hydroxyornithine.

subalignments at $50 \mathrm{bp}$ intervals, revealed increased phylogenetic incompatibility between $\mathrm{A}$ domains and the surrounding domains (Fig. 4a). Segregation analysis to examine whether any of the $400 \mathrm{bp}$ subalignments cluster by substrate specificity identified the region of the A domain that exhibited the greatest phylogenetic incompatibility as clustering strongly by substrate specificity (Fig. 4b).

The TreeOrderScan analysis is consistent with A domain substitution driving NRPS evolution but does not identify the recombination points that have arisen most commonly during natural substitution events. To identify hotspots of recombination, sequence analysis was performed using RDP4, an ensemble of tools that collectively identify regions at which DNA sequences are likely to have recombined ${ }^{33}$. The breakpoint distribution identified recombination hotspots located between $\mathrm{C}$ and $\mathrm{A}$ domains (Fig. 4c, red shading), upstream to the $\mathrm{A}$ domain substrate binding pocket between the A2 and A4 motifs (Fig. 4c, green shading), and downstream to the binding pocket starting from close to the A5 motif (Fig. 4c, blue shading). The largest hotspots were located immediately on either side of the binding pocket, flanking the region that segregates most strongly by substrate specificity (Fig. $4 \mathrm{a}-\mathrm{c}$ ). These data were consistent for each of the Pseudomonas, Bacillus and Streptomyces genera, and inconsistent with the hypothesis that $\mathrm{C}$ and $\mathrm{A}$ domains co-evolve. We conclude that complete or partial A domain substitution appears to play a primary role in diversification of NRPS pathways in nature, rather than being an exception.
Partial A domain substitution has previously been attempted in two key laboratory studies ${ }^{34,35}$. However, only one of these (working in an initiation module that lacks a C domain) described the formation of a modified peptide, the rate of formation of which was greatly reduced in vitro and not tested further in vivo ${ }^{35}$. We tested partial A domain substitution in PvdD using boundaries suggested by our recombination analysis, but did not achieve visible production of pyoverdine in any instance (Supplementary Figs. 11, 12). Reasoning that this might be due to structural clashes restricting efficient recombination of NRPS enzymes within certain domain regions, we used SCHEMA $^{36}$ to predict the number of perturbations generated by recombination of the C-A-T domains from PvdD with the CA-T domains from alternative pyoverdine NRPS modules (Fig. 4d). Whereas the recombination sites employed in our successful linker + A domain substitutions were within regions with low potential to cause structural perturbations during substitution, the recombination hotspot between the A2 and A4 motifs (Fig. 4c, green shading), was in a region with high potential for perturbations. Testing of additional partial A domain substitution constructs that used alternative upstream recombination points in conjunction with the downstream site used in our A domain experiments was also unsuccessful (Supplementary Figs. S11, S12). We believe that the SCHEMA analysis explains why our (and previous ${ }^{34,35}$ ) experimental attempts at partial A domain substitution were generally unsuccessful, or at best highly inefficient ${ }^{35}$. In contrast, because 
a Phylogenetic incompatibility per sequence

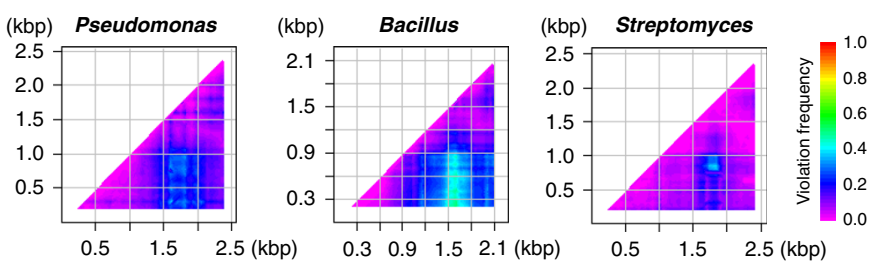

b Segregation by consensus substrate specificity
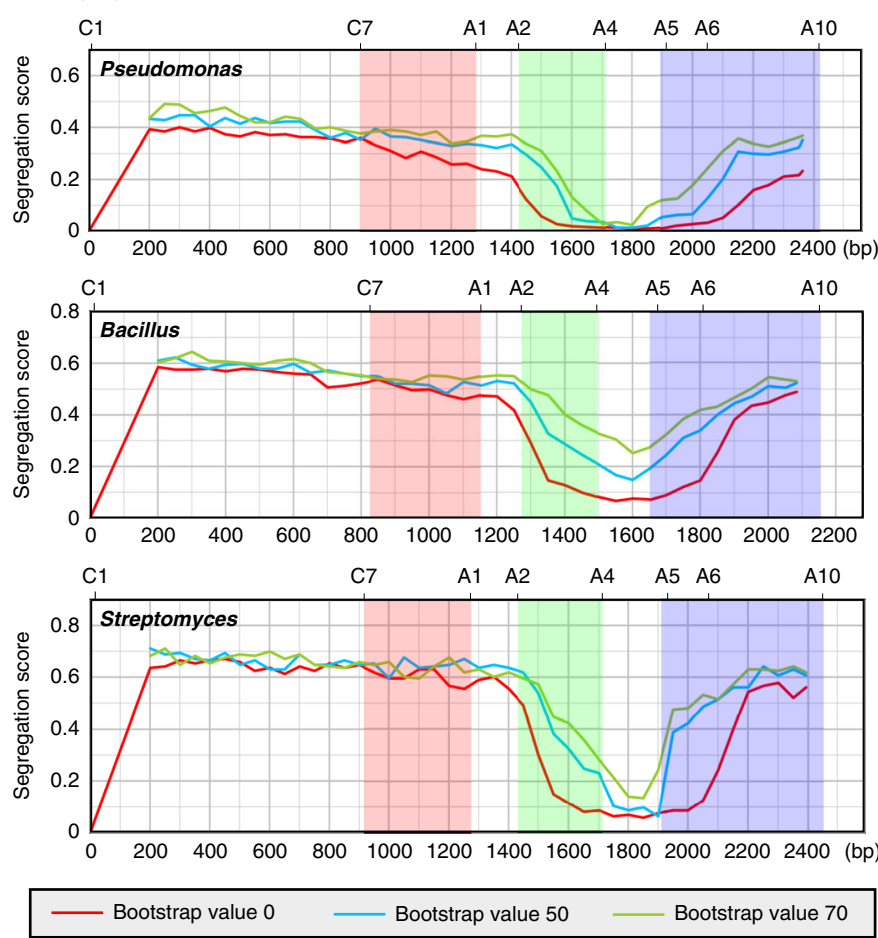

C
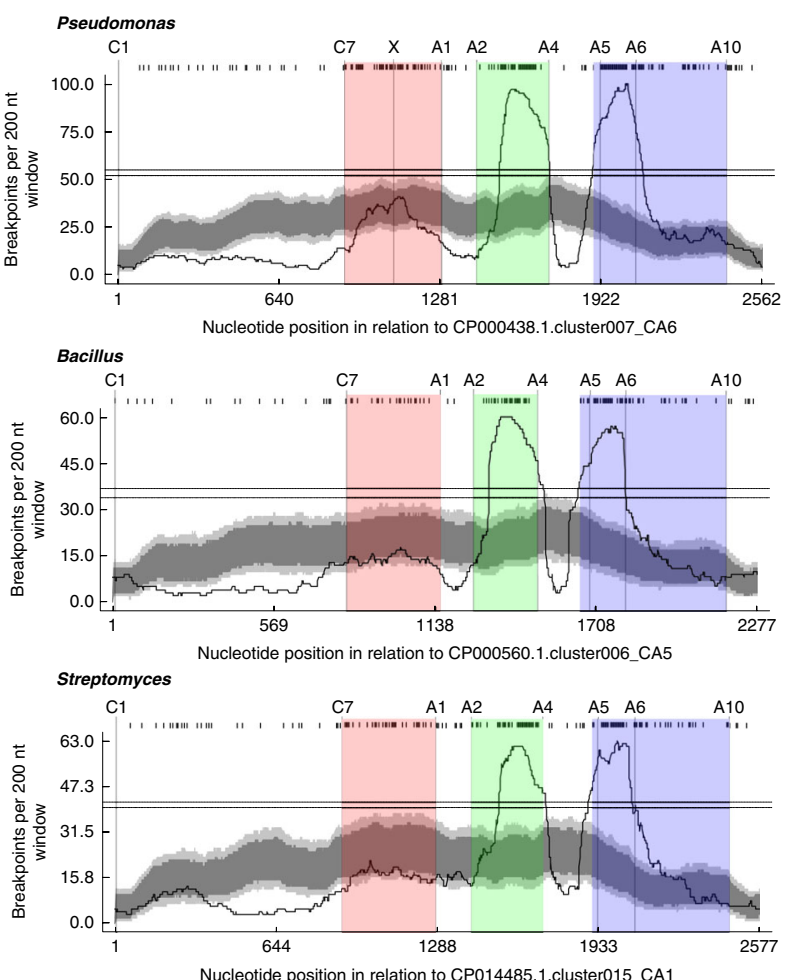

d Clashes at each recombination point

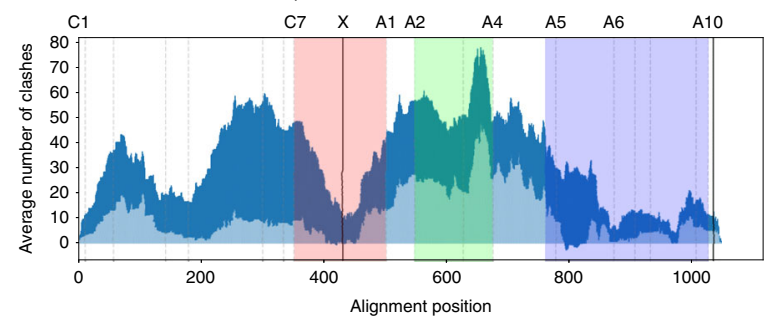

Fig. 4 Natural NRPS diversification is driven primarily by A domain and subdomain substitution. a Phylogenetic compatibility matrices of alignments of C-A-T domains from Pseudomonas, Bacillus and Streptomyces species showing frequencies of phylogeny violations for each pairwise comparison of sequence fragments. Analysis was performed using TreeOrder Scan 31,32 , considering alignments of 400 bp at 50 bp intervals and using a bootstrap value of $70 \%$ to calculate phylogenetic violations. b Segregation of alignments by consensus substrate specificity predictions from antiSMASH ${ }^{22}$. Segregation scores were calculated using a 0\% (red line), 50\% (blue line) and 70\% (green line) bootstrap cut off. The locations of key conserved motifs are indicated along the top of the graph. For this analysis, $400 \mathrm{bp}$ alignments were compared for segregation into groups based on consensus substrate specificity predictions from antiSMASH ${ }^{22}$. A segregation score of 0 means perfect segregation by substrate specificity and a score of 1.0 means no segregation on the basis of substrate specificity. Shaded blocks have been added to aid comparison between the corresponding regions of interest in $\mathbf{b}-\mathbf{d}$. $\mathbf{c}$ Recombination hotspot analysis of C-A-T domains from Pseudomonas, Bacillus and Streptomyces species. ' $X$ ' marks the location of the recombination point used for the successful (linker control) Lys A domain substitution in Fig. 2e. Dark and light grey areas indicate local breakpoint hotspots at the 95 and $99 \%$ confidence level, respectively, and the two horizontal lines indicate cut-offs for global breakpoint hotspots at the 95 and $99 \%$ confidence level. $\mathbf{d}$ The light blue shaded plot indicates the mean number of clashes calculated using SCHEMA that would be introduced by recombination of the nine alternative pyoverdine NRPS modules depicted in Fig. $3 \mathrm{~b}$ with the domains from Pa11 ( $n=9 \mathrm{C}-\mathrm{A}-\mathrm{T}$ domains and the dark blue region indicates \pm SD). Source data are available via the links provided in the "Methods".

natural recombination processes favour short sequences ${ }^{37,38}$, this appears to be a preferred region of recombination during natural NRPS evolution, with the low success rate presumably offset by the high frequency of recombination events. Irrespective, our diverse analyses provide unanimous support for partial or complete A domain substitution being a primary mechanism for NRPS diversification.

A domain substitution in the PheATE-ProCAT model system. Our high success rates in modifying pyoverdine via A domain substitution and our bioinformatics analyses suggested that $\mathrm{C}$ domain acceptor substrate specificity is less of a barrier to NRPS engineering than previously proposed by several groups, including $\mathrm{us}^{3,4,10-13,39}$. However, the possibility remained that the $\mathrm{Pa} 8$ and Pa11 C domains are more relaxed than $\mathrm{C}$ domains such as that in module 2 of tyrocidine biosynthesis-the system that Belshaw et al. ${ }^{3}$ used to develop the original hypothesis of $\mathrm{C}$ domains exhibiting strong acceptor substrate specificity. We therefore considered it important to test whether we could create novel dipeptides by performing A domain substitution within the same system.

Belshaw et al showed that tyrocidine module one (PheATE, incorporating D-Phe) and module two (ProCAT, incorporating 
L-Pro) could be artificially loaded with different amino acids in vitro, and the resulting dipeptide purified and analysed ${ }^{3}$. Artificial loading with L-Pro resulted in the expected product, but no product resulted when ProCAT was loaded with L-Leu, suggesting stringent substrate specificity during condensation. If this hypothesis is true, the C-domain of ProCAT will not accept L-Leu. We therefore considered that efficient production of DPhe-L-Leu dipeptides via A domain substitution in the ProCAT module would disprove the $\mathrm{C}$ domain substrate specificity hypothesis in this foundational model. To avoid potential for in vitro inconsistencies and facilitate construct generation and analysis we used a similar two-plasmid in vivo system to previous researchers ${ }^{40,41}$, with the replacement of the $\mathrm{T}$ domain from ProCAT with the T-Te domains from SrfC, to enable release of linear D-Phe-L-Leu dipeptides ${ }^{42,43}$. The genetic regions encoding four different Leu-specifying A domains were selected for substitution into the ProCATTe plasmid and compared to an unsubstituted control (Supplementary Table S2; Supplementary Fig. S13). All A domains shared relatively low amino acid identity to the A domain from ProCAT (40.4\% to 47.6\%; Supplementary Table 2). The Leu-specifying A domain from SrfC was of particular interest because the crystal structure of this module fuelled speculation that C-A domains form a tight interface, which may further restrict A domain substitution ${ }^{21}$. As such, this experiment combined all the main factors that have been suggested to prohibit effective A domain substitution, i.e. a C domain believed to exhibit tight acceptor site specificity, the substitution of distantly related A domains, and substitution of an A domain believed to depend on a tight $\mathrm{C}$-A domain interface with its cognate $\mathrm{C}$ domain partner.

The recombinant ProCATTe plasmids and a second plasmid containing PheATE were used to co-transform a BAP1 strain of E. coli ${ }^{44}$. The strains were grown for $18 \mathrm{~h}$, after which the supernatant was extracted and dipeptides quantified using HPLC and absorbance at $214 \mathrm{~nm}$ (Fig. 5). We detected production of the native D-Phe-L-Pro diketopiperazine at $7.8 \mathrm{mg} / \mathrm{L}$ by the control strain (Fig. 5a), a yield that compares favourably to previous reports ${ }^{40,41}$. We also detected D-Phe-L-Leu dipeptides for three of the four strains containing Leu-specific A domain substitutions, at yields ranging from ca. 25 to $40 \%$ of the D-Phe-L-Pro control (Fig. 5b). Despite sharing the lowest amino acid identity with the ProCAT A domain, and its previously hypothesised requirement to maintain a native $\mathrm{C}$ domain interface, the strain containing the A domain from SrfC was found to produce D-Phe-L-Leu at 1.8 $\mathrm{mg} / \mathrm{L}$. We would not have expected these successful outcomes based on the previous work of Belshaw et al. ${ }^{3}$, and conclude stringent acceptor site specificity against Leu as previously inferred for this $\mathrm{C}$ domain is not a barrier to creating successful A domain substitutions. Rather, it appears that successful A domain substitution relies greatly on the recombination boundaries used.

\section{Discussion}

While natural evolution has given rise to a large diversity of nonribosomal peptides, effective re-engineering of NRPS templates in the laboratory has proven difficult $13,19,45$. A primary focus in reengineering NRPS enzymes has been to accommodate the presumed acceptor site specificity of the $\mathrm{C}$ domain. We have shown this might not be as necessary as previously thought. Our identification of tolerant recombination points via an unbiased DNA shuffling approach allowed us to subsequently produce modified products with high success rates in two diverse NRPS systems, one of which should have been intractable, as the cornerstone of the C-domain acceptor site proof-reading hypothesis. The possibility remains that both systems might contain relaxed- specificity C domains, and other subtypes of $\mathrm{C}$ domains might exist that demonstrate stringent acceptor site specificity. Nevertheless, we have also substantially expanded upon previous work that has examined NRPS evolution in individual pathways by providing evidence across diverse genera that NRPS diversification occurs predominantly by A domain (or subdomain) substitution. This observation is inconsistent with widespread acceptor site specificity existing as a barrier to successful A domain substitution. Further work will be needed to understand how broadly these findings can be applied, but we consider they hold considerable potential for efficient rational and combinatorial improvement of medically and industrially relevant peptides.

\section{Methods}

DNA manipulation. All plasmids, primers and sequences used in this study are provided in the Supplementary file Supplementary Data 1 - Plasmids Primers and Gene Sequences.xlsx.

To create vectors for substituting domains into $p v d D$, the $\mathrm{P}_{\mathrm{BAD}}$ promoter was excised from pSW196 using the restriction sites NsiI and SacI and ligated into pUCP22 using the restriction sites PstI and SacI. The resulting plasmid was named pUCBAD. Next, the $p v d D$ gene lacking the C-A domains from the second module (module Pa11) was excised from the plasmid $\mathrm{pSMC}^{9}$ using NheI and SacI restriction sites and annealed into the pUCBAD vector using the restriction sites NheI and SacI to create the vector pUCBAD-SMC. The Thr-specific A domain from the second module of PvdD and the Lys-specific A domain from the first module of PvdJ (module Pa8) were PCR amplified and separately ligated into the pUCBAD-SMC vector. This resulted in the creation of the vectors pDEC-Thr and pDEC-Lys, which contained a Thr-specific A domain and a Lys- specific A domain variant of the $p v d D$ gene, respectively. For modifying the third variable region of the $\mathrm{C}$ domain from $p v d D$, the first two variable regions of the $p v d D \mathrm{C}$ domain from module Pa11 were PCR amplified. The resulting fragment was ligated into the pDEC-Thr vector using a $5^{\prime} \mathrm{SpeI} / \mathrm{XbaI}$ and a $3^{\prime}$ SalI/SalI ligation to create the plasmid pTRN. The destruction of the SpeI restriction site within the vector by SpeI/XbaI ligation meant the introduced SpeI site downstream to region 2 of the $\mathrm{C}$ domain was unique within the plasmid, allowing insertion of region 3 using SpeI and SalI restriction sites.

C domains were created by overlap PCR or synthesis (Twist Bioscience; San Francisco, CA). C domain sequences were amplified using the appropriate forward and reverse primers specific to the C-domain from modules Pa8-Lys, Pa11-Thr, Ps5-Ser or Pf6-Orn (Pa indicates Pseudomonas aeruginosa PA01; Ps5-Ser indicates the fifth serine-incorporating pyoverdine NRPS module from Pseudomonas syringae 1448a; Pf6-Orn indicates the sixth ornithine-incorporating pyoverdine NRPS module from Pseudomonas fluorescens SBW25; Supplementary Fig. S1). PCR products were digested using XbaI and XhoI, and ligated into the plasmids pDECThr and pDEC-Lys using compatible SpeI and SalI restriction sites. The partial C domain fragments containing region 3 of a $\mathrm{C}$ domain were amplified using the appropriate forward and reverse primers. PCR products were digested using SpeI and XhoI, and ligated into the plasmid pTRN at compatible SpeI and SalI restriction sites.

A domains were selected to activate a range of substrates, from modules exhibiting a range of amino acid sequence identities with Pal1-Thr

(Supplementary Table S1). To enable cloning of A domains into the substitution vector pTRN, inserts were designed to have an upstream region identical to the Cdomain from Pa11-Thr, fused to the linker and A domain from the selected modules. Recombination points for each substitution are shown in Supplementary Fig. S9. To reduce the GC content to acceptable levels for synthesis, 5_AP013068.1 cluster003_CA1 was codon optimised for P. aeruginosa PAO1 using the guided random method in OPTIMIZER ${ }^{46}$

Partial A domain substitutions were created for the Lys-specific A domain from module Pa8 and the Ser-specific A domain from number 2 in Fig. 2B. Substitutions were created by ligating synthetic DNA constructs into the vector pTRN. Recombination points for partial A domain substitutions are shown in Supplementary Fig. S11, and are labelled in the Supplementary file Supplementary Data 1 - Plasmids Primers and Gene Sequences.xlsx using the GrsA nomenclature from Kries et al. ${ }^{35}$. The recombination points tested were T221 and I352, corresponding to those used previously by Kries et al. ${ }^{35}$, and K205 and A322, corresponding to those used by Crüsemann et al. ${ }^{34}$ Based on our SCHEMA analysis we also tested the promising recombination points S233 and A332 as well as upstream recombination points at A185, I167 and S233 in combination with the downstream site used in the full A domain substitutions.

C-A domains from modules Ps5-Ser, Pf6-Orn and Pa8-Lys were amplified by PCR and ligated into the vector pUCBAD-SMC.

DNA encoding PheATE and ProC-TTe was artificially synthesised (Twist Bioscience; San Francisco, CA) following codon optimisation for E. coli using the guided random method in OPTIMIZER ${ }^{46}$. PheATE was cloned into pACYCDuet- 1 using NcoI and XhoI restriction sites, and ProC-TTe was cloned into pET28a+ 
a
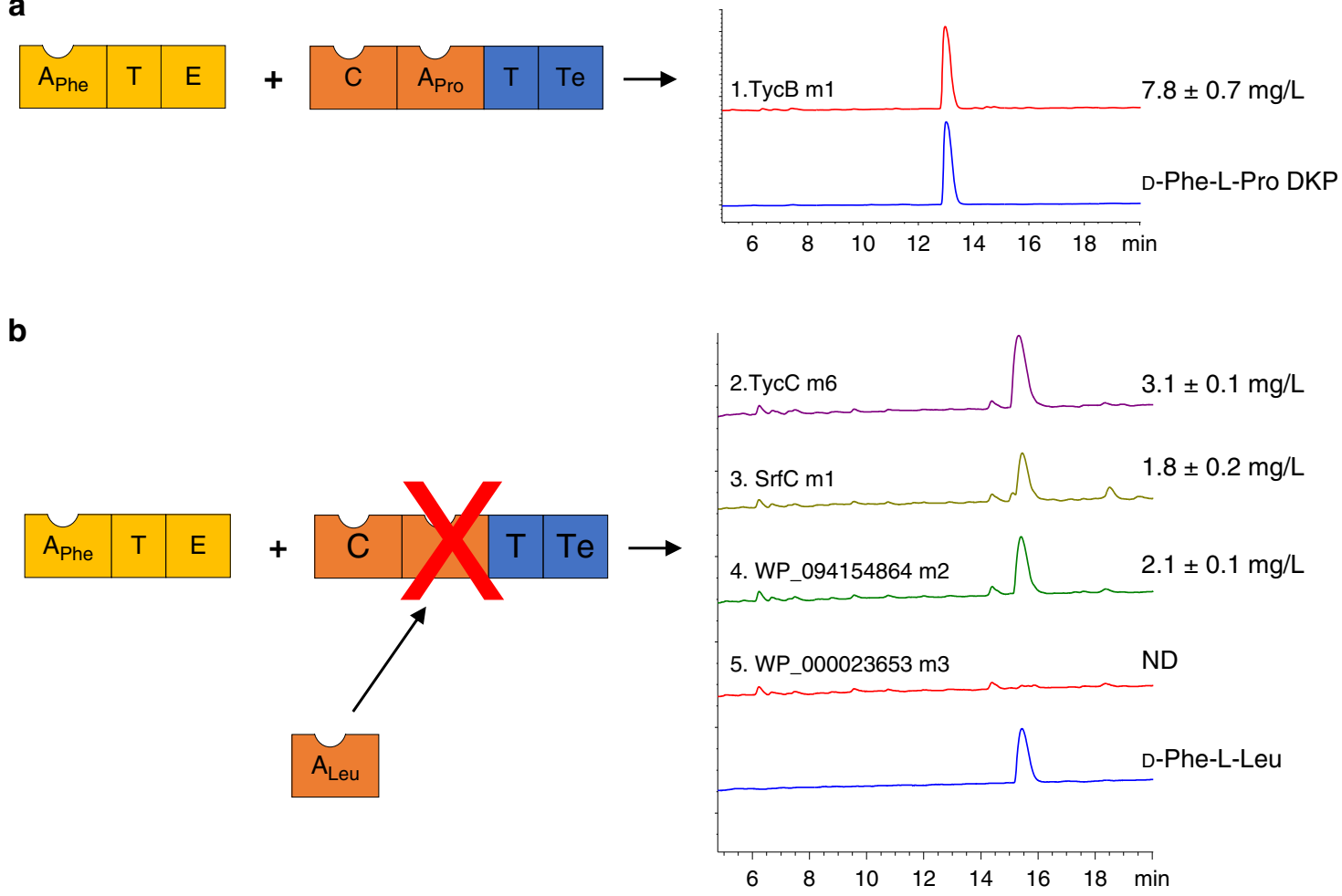

Fig. 5 Successful substitution of Leu-specifying A domains in the PheATE-ProCAT model system. a Schematic showing the domain arrangement of the PheATE/ProCATTe constructs used in this study and HPLC traces comparing the product made by an E. coli BAP1 strain expressing these constructs (1) relative to an analytical standard of D-Phe-L-Pro DKP (dark blue trace). b Schematic and HPLC traces for the products generated by four strains (2-5) bearing variants of ProCATTe in which the Pro-specifying A domain had been substituted by a Leu-specifying A domain. Strains 2,3 and 4 show a peak corresponding to an analytical standard of D-Phe-L-Leu (dark blue trace) ( $n=3$ independent experiments and data are presented as the mean yield \pm SD). Masses of the expected products were verified using HR-ESI-MS (Supplementary Fig. S14). Source data are provided as a Source Data file.

using NcoI and XhoI restriction sites. The Pro-specific A domain from ProCAT and four Leu-specific A domains were codon optimised and ligated into the SpeI and NotI restriction sites of ProC-TTe using compatible NheI and NotI sites. Alignments of A domains and sequence origins and identities are provided in Supplementary Fig. S14 and Supplementary Table S2).

Data analysis. Structural models of the $\mathrm{C}$ domain from the second module of PvdD were created by submitting the $C$ domain sequence to multiple automated servers ${ }^{47-62}$, and using the Swiss-Model server (http://swissmodel.expasy.org// ${ }^{63}$ and Modeller 9.1164. Models created from each method were submitted to the QMEAN server to obtain QMEAN6 and QMEANclust scores ${ }^{65,66}$. The model RaptorXmsa was selected to work with as it scored best overall considering both measurements. The model RaptorXmsa aligned well to the $\mathrm{C}$ domain structure from $\mathrm{TycC}^{21}$ with a root mean-square deviation for the backbone $a$ carbons of $0.381 \AA$.

The antiSMASH database ${ }^{22}$ was queried for all NRPS biosynthetic gene clusters from the genera Pseudomonas, Bacillus and Streptomyces. The genbank files for each cluster were downloaded, and the python script extractCATdomains_consensus. py (available at https://github.com/MarkCalcott/NRPS_evolution/tree/master/ Raw_sequences) used to find, extract and save DNA encoding ${ }^{\mathrm{L}} \mathrm{C}_{\mathrm{L}}-\mathrm{A}-\mathrm{T}$ tridomains into a separate FASTA file for each genera. The criteria for extracting tridomains were that the A domain had an antiSMASH consensus substrate specificity prediction, and that C-A-T domains were located on the same protein in the correct order, with fewer than 250 amino acid residues between domains. Each extracted DNA sequence was annotated with the consensus substrate specificity. Sequences were dereplicated using USEARCH $10.0 .240^{67}$, and clustered at $95 \%$ identity (Supplementary Table S3). A codon-alignment of the centroid nucleotide sequence from each cluster was generated using MUSCLE ${ }^{68}$. Sequences were trimmed to the $\mathrm{Cl}$ and $\mathrm{T}$ motifs inclusive, and any sequences not containing these motifs were removed. Regions of ambiguous alignment were removed using GBLOCK version $0.91 b^{69}$. The default parameters were used for GBLOCK except the minimum number of sequences for a flank position was set equal to $50 \%$ of the total sequences, the minimum length of a block was five, and gap positions were allowed in half of the sequences.

Simple sequence editor version 1.3 was used for TreeOrder scan analysis ${ }^{31,32}$ Sequences were grouped by the antiSMASH consensus prediction of A domain substrate specificity. Length of fragments were $400 \mathrm{bp}$ with an increment of $50 \mathrm{bp}$, and 100 bootstraps. The random seed was set to 1 . To detect recombination hot spots, the aligned sequences were analysed using RDP4 ${ }^{33}$. Default settings were used except sequences were specified as linear, only recombination events detected by at least three methods were considered and alignment consistency was unchecked. A breakpoint distribution plot was created using a 200 bp window and 1000 permutations.

The structure 2VSQ was used as it was identified as the top template for modelling the CAT-domains from Pa11 using the Swiss-Model server ${ }^{63}$. Sequences were aligned using MUSCLE ${ }^{68}$ and then SCHEMA ${ }^{36}$ was used to create a contact map for each structure. The python script Schema_profile.py (available at https:// github.com/MarkCalcott/NRPS_evolution/tree/master/Schema_Bar_Graph) calculated the average number of clashes using SCHEMA for each recombination point between Pall and the modules used as a source of A-domains.

Analysing pyoverdine production. Each strain to be analysed was first used to inoculate $200 \mu \mathrm{L}$ of low salt LB in a 96 well plate. After $24 \mathrm{~h}$ growth at $37^{\circ} \mathrm{C}, 10 \mu \mathrm{L}$ of each starter culture was used to inoculate $190 \mu \mathrm{L}$ of M9 media amended with $0.1 \%(\mathrm{w} / \mathrm{v}) \mathrm{L}$-arabinose and $4 \mathrm{~g} / \mathrm{L}$ succinate $(\mathrm{pH}$ 7.0). Cultures were grown for $37^{\circ} \mathrm{C}$ for $24 \mathrm{~h}$, centrifuged to pellet bacteria, and then $100 \mu \mathrm{L}$ of supernatant transferred to a fresh 96 well plate and diluted twofold in fresh M9 media to give a total volume of $200 \mu \mathrm{L}$. Absorbance ( $400 \mathrm{~nm}$ ) was measured using an EnSpire 2300 Multilabel Reader (PerkinElmer, Waltham, MA, USA). For mass spectrometry analysis, $1 \mu \mathrm{L}$ of supernatant was mixed with $20 \mu \mathrm{L}$ of matrix ( $500 \mu \mathrm{L}$ acetonitrile, $500 \mu \mathrm{L}$ ultrapure water, $1 \mu \mathrm{L}$ trifluoroacetic acid, $10 \mu \mathrm{g} \alpha$-cyano-4-hydroxycinnamic acid) ${ }^{16,70}$. Aliquots of $0.5 \mu \mathrm{L}$ were spotted in triplicate onto an Opti-TOF 384 well MALDI plate (Applied Biosystems, Foster City, CA) and allowed to dry at room temperature. Spots were analysed using a MALDI TOF/TOF 5800 mass spectrometer (Applied Biosystems) in positive ion mode. Peaks were externally calibrated using cal2 calibration mixture (Applied Biosystems). Peaks in spectra were labelled in Data Explorer (Applied Biosystems).

Analysing dipeptide production. The plasmids containing PheATE and ProCATTe were transformed into the E. coli strain BAP1 ${ }^{44}$. A $100 \mu \mathrm{L}$ aliquot from an overnight culture was used to inoculate $10 \mathrm{~mL}$ M9 media amended with $0.1 \%(\mathrm{w} / \mathrm{v})$ casamino acids and $0.4 \%(\mathrm{w} / \mathrm{v}) \mathrm{D}$-glucose. The culture was grown at $30^{\circ} \mathrm{C}$ for $5 \mathrm{~h}$ and then incubated on ice for $20 \mathrm{~min}$. IPTG was added to a final concentration of 
$1 \mathrm{mM}$, and the culture grown for a further $16 \mathrm{~h}$ at $18^{\circ} \mathrm{C}$. The cells were pelleted by centrifugation at $2000 \mathrm{~g}$ for $20 \mathrm{~min}$. The supernatant was transferred to a fresh tube and immediately extracted with 4:1 butanol-chloroform (vol/vol). The organic layer was washed with 1 volume of $0.1 \mathrm{M} \mathrm{NaCl}$ and the aqueous phase removed. Solvent was removed under vacuum and residue dissolved in $150 \mu \mathrm{L}$ of $10 \%$ $\mathrm{CH}_{3} \mathrm{CN}$. HPLC separation was performed on an Agilent $1200 \mathrm{LC}$ system using a C18 reverse-phase column (PhenoSphere-Next, 150 by $4.6 \mathrm{~mm}$; pore size, $120 \AA$; particle size, $3 \mu \mathrm{m}$; Phenomenex, Torrance, CA). The column oven was set at $40^{\circ} \mathrm{C}$ and injection volume at $30 \mu \mathrm{L}$. Elution was conducted with a mobile phase consisting of (A) water $+0.1 \%$ formic acid, and $(B) \mathrm{CH}_{3} \mathrm{CN}+0.1 \%$ formic acid. Following $1 \mathrm{~min}$ at $10 \% \mathrm{~B}$, a gradient up to $25 \% \mathrm{~B}$ was performed in $17 \mathrm{~min}$. The flow rate was set at $1 \mathrm{~mL} / \mathrm{min}$. Concentrations were determined by comparison to chemically synthesised standards with $98 \%$ purity by LifeTein (South Plainfield, NJ). For HPLC-MS, separation was performed on an Agilent 6530 Accurate-Mass Q-TOF LC/MS system equipped with an Agilent 1260 HPLC system (Agilent, Santa Clara, CA). The same HPLC conditions as previously were used except the column oven was set at $45^{\circ} \mathrm{C}$ and injection volume at $1 \mu \mathrm{L}$

Reporting summary. Further information on research design is available in the Nature Research Reporting Summary linked to this article.

\section{Data availability}

Source data are provided with this paper.

\section{Code availability}

All code generated during this study is available on GitHub via the following links: (1) python script extractCATdomains_consensus.py https:/github.com/MarkCalcott/ NRPS_evolution/tree/master/Raw_sequences (2) python script Schema_profile.py https://github.com/MarkCalcott/NRPS_evolution/tree/master/Schema_Bar_Graph.

Received: 5 June 2020; Accepted: 19 August 2020;

Published online: 11 September 2020

\section{References}

1. Stachelhaus, T., Schneider, A. \& Marahiel, M. A. Rational design of peptide antibiotics by targeted replacement of bacterial and fungal domains. Science 269, 69-72 (1995)

2. Schneider, A., Stachelhaus, T. \& Marahiel, M. A. Targeted alteration of the substrate specificity of peptide synthetases by rational module swapping. Mol. Gen. Genet. 257, 308-318 (1998)

3. Belshaw, P. J., Walsh, C. T. \& Stachelhaus, T. Aminoacyl-CoAs as probes of condensation domain selectivity in nonribosomal peptide synthesis. Science 284, 486-489 (1999).

4. Ehmann, D. E., Trauger, J. W., Stachelhaus, T. \& Walsh, C. T. AminoacylSNACs as small-molecule substrates for the condensation domains of nonribosomal peptide synthetases. Chem. Biol. 7, 765-772 (2000)

5. Linne, U. \& Marahiel, M. A. Control of directionality in nonribosomal peptide synthesis: role of the condensation domain in preventing misinitiation and timing of epimerization. Biochemistry 39, 10439-10447 (2000).

6. Mootz, H. D., Schwarzer, D. \& Marahiel, M. A. Construction of hybrid peptide synthetases by module and domain fusions. Proc. Natl Acad. Sci. U.S.A. 97, 5848-5853 (2000).

7. Duerfahrt, T., Doekel, S., Sonke, T., Quaedflieg, P. J. \& Marahiel, M. A. Construction of hybrid peptide synthetases for the production of alpha-laspartyl-1-phenylalanine, a precursor for the high-intensity sweetener aspartame. Eur. J. Biochem. 270, 4555-4563 (2003).

8. Baltz, R. H. Combinatorial biosynthesis of cyclic lipopeptide antibiotics: a model for synthetic biology to accelerate the evolution of secondary metabolite biosynthetic pathways. ACS Synth. Biol. 3, 748-758 (2014).

9. Calcott, M. J., Owen, J. G., Lamont, I. L. \& Ackerley, D. F. Biosynthesis of novel pyoverdines by domain substitution in a nonribosomal peptide synthetase of Pseudomonas aeruginosa. Appl. Environ. Microbiol. 80, 5723-5731 (2014).

10. Bozhuyuk, K. A. J. et al. De novo design and engineering of non-ribosomal peptide synthetases. Nat. Chem. 10, 275-281 (2018).

11. Bozhuyuk, K. A. J. et al. Modification and de novo design of non-ribosomal peptide synthetases using specific assembly points within condensation domains. Nat. Chem. 11, 653-661 (2019).

12. Steiniger, C., Hoffmann, S. \& Sussmuth, R. D. Desymmetrization of cyclodepsipeptides by assembly mode switching of iterative nonribosomal peptide synthetases. ACS Synth. Biol. 8, 661-667 (2019).

13. Bozhuyuk, K. A., Micklefield, J. \& Wilkinson, B. Engineering enzymatic assembly lines to produce new antibiotics. Curr. Opin. Microbiol. 51, 88-96 (2019).
14. Ackerley, D. F., Caradoc-Davies, T. T. \& Lamont, I. L. Substrate specificity of the nonribosomal peptide synthetase PvdD from Pseudomonas aeruginosa. J. Bacteriol. 185, 2848-2855 (2003).

15. Ackerley, D. F. \& Lamont, I. L. Characterization and genetic manipulation of peptide synthetases in Pseudomonas aeruginosa PAO1 in order to generate novel pyoverdines. Chem. Biol. 11, 971-980 (2004).

16. Calcott, M. J. \& Ackerley, D. F. Portability of the thiolation domain in recombinant pyoverdine non-ribosomal peptide synthetases. BMC Microbiol. 15, 162 (2015).

17. Bloudoff, K., Alonzo, D. A. \& Schmeing, T. M. Chemical probes allow structural insight into the condensation reaction of nonribosomal peptide synthetases. Cell Chem. Biol. 23, 331-339 (2016).

18. Rausch, C., Hoof, I., Weber, T., Wohlleben, W. \& Huson, D. H. Phylogenetic analysis of condensation domains in NRPS sheds light on their functional evolution. BMC Evol. Biol. 7, 78 (2007).

19. Sussmuth, R. D. \& Mainz, A. Nonribosomal peptide synthesis-principles and prospects. Angew. Chem. Int. Ed. 56, 3770-3821 (2017).

20. Samel, S. A., Schoenafinger, G., Knappe, T. A., Marahiel, M. A. \& Essen, L. O. Structural and functional insights into a peptide bond-forming bidomain from a nonribosomal peptide synthetase. Structure 15, 781-792 (2007).

21. Tanovic, A., Samel, S. A., Essen, L. O. \& Marahiel, M. A. Crystal structure of the termination module of a nonribosomal peptide synthetase. Science 321, 659-663 (2008).

22. Blin, K., Medema, M. H., Kottmann, R., Lee, S. Y. \& Weber, T. The antiSMASH database, a comprehensive database of microbial secondary metabolite biosynthetic gene clusters. Nucleic Acids Res. 45, D555-D559 (2017).

23. Baltz, R. H. Synthetic biology, genome mining, and combinatorial biosynthesis of NRPS-derived antibiotics: a perspective. J. Ind. Microbiol. Biotechnol. 45, 635-649 (2018)

24. Lautru, S. \& Challis, G. L. Substrate recognition by nonribosomal peptide synthetase multi-enzymes. Microbiol-SGM 150, 1629-1636 (2004).

25. Fewer, D. P. et al. Recurrent adenylation domain replacement in the microcystin synthetase gene cluster. BMC Evol. Biol. 7, 183 (2007)

26. Ishida, K. et al. Plasticity and evolution of aeruginosin biosynthesis in cyanobacteria. Appl. Environ. Microbiol. 75, 2017-2026 (2009).

27. Hofer, I. et al. Insights into the biosynthesis of hormaomycin, an exceptionally complex bacterial signaling metabolite. Chem. Biol. 18, 381-391 (2011).

28. Götze, S. et al. Structure elucidation of the syringafactin lipopeptides provides insight in the evolution of nonribosomal peptide synthetases. Chem. Sci. 10, 10979-10990 (2019).

29. Meyer, S. et al. Biochemical dissection of the natural diversification of microcystin provides lessons for synthetic biology of NRPS. Cell Chem. Biol. 23, 462-471 (2016).

30. Li, R. F., Oliver, R. A. \& Townsend, C. A. Identification and characterization of the sulfazecin monobactam biosynthetic gene cluster. Cell Chem. Biol. 24 24-34 (2017).

31. Simmonds, P. SSE: a nucleotide and amino acid sequence analysis platform. BMC Res. Notes 5, 50 (2012).

32. Simmonds, P. Recombination and selection in the evolution of picornaviruses and other mammalian positive-stranded RNA viruses. J. Virol. 80, 11124-11140 (2006)

33. Martin, D. P., Murrell, B., Golden, M., Khoosal, A. \& Muhire, B. RDP4: Detection and analysis of recombination patterns in virus genomes. Virus Evol. 1, vev003 (2015)

34. Crusemann, M., Kohlhaas, C. \& Piel, J. Evolution-guided engineering of nonribosomal peptide synthetase adenylation domains. Chem. Sci. 4, 1041-1045 (2013)

35. Kries, H., Niquille, D. L. \& Hilvert, D. A subdomain swap strategy for reengineering nonribosomal peptides. Chem. Biol. 22, 640-648 (2015).

36. Voigt, C. A., Martinez, C., Wang, Z. G., Mayo, S. L. \& Arnold, F. H. Protein building blocks preserved by recombination. Nat. Struct. Mol. Biol. 9, 553-558 (2002).

37. Gonzalez-Torres, P., Rodriguez-Mateos, F., Anton, J. \& Gabaldon, T. Impact of homologous recombination on the evolution of prokaryotic core genomes. Mbio 10, pii: ARTN e02494-18 (2019).

38. Didelot, X. \& Maiden, M. C. J. Impact of recombination on bacterial evolution. Trends Microbiol. 18, 315-322 (2010)

39. Brown, A. S., Calcott, M. J., Owen, J. G. \& Ackerley, D. F. Structural, functional and evolutionary perspectives on effective re-engineering of nonribosomal peptide synthetase assembly lines. Nat. Prod. Rep. 35, 1210-1228 (2018).

40. Gruenewald, S., Mootz, H. D., Stehmeier, P. \& Stachelhaus, T. In vivo production of artificial nonribosomal peptide products in the heterologous host Escherichia coli. Appl. Environ. Microbiol. 70, 3282-3291 (2004).

41. Kries, $H$. et al. Reprogramming nonribosomal peptide synthetases for "Clickable" amino acids. Angew. Chem. Int. Ed. 53, 10105-10108 (2014).

42. Schwarzer, D., Mootz, H. D. \& Marahiel, M. A. Exploring the impact of different thioesterase domains for the design of hybrid peptide synthetases. Chem. Biol. 8, 997-1010 (2001). 
43. Hahn, M. \& Stachelhaus, T. Harnessing the potential of communicationmediating domains for the biocombinatorial synthesis of nonribosomal peptides. Proc. Natl Acad. Sci. USA 103, 275-280 (2006).

44. Pfeifer, B. A., Admiraal, S. J., Gramajo, H., Cane, D. E. \& Khosla, C. Biosynthesis of complex polyketides in a metabolically engineered strain of $E$. coli. Science 291, 1790-1792 (2001).

45. Winn, M., Fyans, J. K., Zhuo, Y. \& Micklefield, J. Recent advances in engineering nonribosomal peptide assembly lines. Nat. Prod. Rep. 33, 317-347 (2016).

46. Puigbo, P., Guzman, E., Romeu, A. \& Garcia-Vallve, S. OPTIMIZER: a web server for optimizing the codon usage of DNA sequences. Nucleic Acids Res. 35, W126-W131 (2007).

47. Bates, P. A., Kelley, L. A., MacCallum, R. M. \& Sternberg, M. J. E. Enhancement of protein modeling by human intervention in applying the automatic programs 3D-JIGSAW and 3D-PSSM. Proteins Suppl 5, 39-46 (2001).

48. Nielsen, M., Lundegaard, C., Lund, O. \& Petersen, T. N. CPHmodels-3.0remote homology modeling using structure-guided sequence profiles. Nucleic Acids Res. 38, W576-W581 (2010)

49. Lambert, C., Leonard, N., De Bolle, X. \& Depiereux, E. ESyPred3D: prediction of proteins 3D structures. Bioinformatics 18, 1250-1256 (2002).

50. Yang, Y. D., Faraggi, E., Zhao, H. Y. \& Zhou, Y. Q. Improving protein fold recognition and template-based modeling by employing probabilistic-based matching between predicted one-dimensional structural properties of query and corresponding native properties of templates. Bioinformatics 27, 2076-2082 (2011).

51. Jones, D. T. et al. Prediction of novel and analogous folds using fragment assembly and fold recognition. Proteins 61, 143-151 (2005).

52. Offman, M. N., Fitzjohn, P. W. \& Bates, P. A. Developing a move-set for protein model refinement. Bioinformatics 22, 1838-1845 (2006).

53. Kallberg, M. et al. Template-based protein structure modeling using the RaptorX web server. Nat. Protoc. 7, 1511-1522 (2012).

54. Shi, J. Y., Blundell, T. L. \& Mizuguchi, K. FUGUE: sequence-structure homology recognition using environment-specific substitution tables and structure-dependent gap penalties. J. Mol. Biol. 310, 243-257 (2001).

55. Rykunov, D., Steinberger, E., Madrid-Aliste, C. J. \& Fiser, A. Improved scoring function for comparative modeling using the M4T method. J. Struct. Funct. Genomics. 10, 95-99 (2009).

56. Zhang, Y. I-TASSER server for protein $3 \mathrm{D}$ structure prediction. BMC Bioinform. 9 https://doi.org/10.1186/1471-2105-9-40 (2008).

57. Zhou, H. Y. \& Skolnick, J. Ab initio protein structure prediction using ChunkTASSER. Biophys. J. 93, 1510-1518 (2007).

58. Wang, Z., Eickholt, J. \& Cheng, J. L. MULTICOM: a multi-level combination approach to protein structure prediction and its assessments in CASP8. Bioinformatics 26, 882-888 (2010).

59. Kelley, L. A. \& Sternberg, M. J. E. Protein structure prediction on the Web: a case study using the Phyre server. Nat. Protoc. 4, 363-371 (2009).

60. Zhang, J. F. et al. MUFOLD: a new solution for protein 3D structure prediction. Proteins 78, 1137-1152 (2010).

61. Zhou, H. \& Skolnick, J. Template-based protein structure modeling using TASSERVMT. Proteins 80, 352-361 (2012)

62. Zhou, H. \& Skolnick, J. Protein structure prediction by pro-Sp3-TASSER. Biophys. J. 96, 2119-2127 (2009).

63. Arnold, K., Bordoli, L., Kopp, J. \& Schwede, T. The SWISS-MODEL workspace: a web-based environment for protein structure homology modelling. Bioinformatics 22, 195-201 (2006).

64. Eswar, N. et al. Comparative protein structure modeling using modeller. Curr. Protoc. Bioinforma. 15, 5.6.1-5.6.30 (2006).

65. Benkert, P., Tosatto, S. C. E. \& Schomburg, D. QMEAN: a comprehensive scoring function for model quality assessment. Proteins 71, 261-277 (2008)

66. Benkert, P., Schwede, T. \& Tosatto, S. C. E. QMEANclust: estimation of protein model quality by combining a composite scoring function with structural density information. BMC Struct. Biol. 9, 35 (2009).
67. Edgar, R. C. Search and clustering orders of magnitude faster than BLAST. Bioinformatics 26, 2460-2461 (2010).

68. Edgar, R. C. MUSCLE: multiple sequence alignment with high accuracy and high throughput. Nucleic Acids Res. 32, 1792-1797 (2004).

69. Castresana, J. Selection of conserved blocks from multiple alignments for their use in phylogenetic analysis. Mol. Biol. Evol. 17, 540-552 (2000).

70. Owen, J. G., Calcott, M. J., Robins, K. J. \& Ackerley, D. F. Generating functional recombinant NRPSenzymes in the laboratory setting via peptidyl carrier protein engineering. Cell Chem. Biol. 23, 1395-1406 (2016).

\section{Acknowledgements}

This work was supported by the Royal Society of New Zealand Marsden Fund (grants 09VUW-01 to D.F.A. and 18-VUW-082 to M.J.C.) and the Health Research Council of New Zealand (grant 16/172 to D.F.A. and J.G.O.). We thank Prof Iain Lamont for his pioneering work in defining the genetics and regulation of pyoverdine biosynthesis and for originally introducing us to this versatile model NRPS system.

\section{Author contributions}

D.F.A. and M.J.C. conceived the work, with additional ideas contributed by J.G.O. M.J.C. performed the experiments, bioinformatics and prepared all figures. J.G.O. provided bioinformatics support. M.J.C. and D.F.A. wrote the manuscript, and all authors approved the final version.

\section{Competing interests}

Drs. Calcott and Ackerley have submitted provisional patent filing AU2019903420, teaching methods for re-engineering NRPS assembly lines based on the optimal A domain recombination sites identified in this research. Dr Owen declares no competing interests.

\section{Additional information}

Supplementary information is available for this paper at https://doi.org/10.1038/s41467020-18365-0.

Correspondence and requests for materials should be addressed to D.F.A.

Peer review information: Nature Communications thanks the anonymous reviewers for their contribution to the peer review of this work. Peer review reports are available.

Reprints and permission information is available at http://www.nature.com/reprints

Publisher's note Springer Nature remains neutral with regard to jurisdictional claims in published maps and institutional affiliations.

\begin{abstract}
cc) (i) Open Access This article is licensed under a Creative Commons BY Attribution 4.0 International License, which permits use, sharing adaptation, distribution and reproduction in any medium or format, as long as you give appropriate credit to the original author(s) and the source, provide a link to the Creative Commons license, and indicate if changes were made. The images or other third party material in this article are included in the article's Creative Commons license, unless indicated otherwise in a credit line to the material. If material is not included in the article's Creative Commons license and your intended use is not permitted by statutory regulation or exceeds the permitted use, you will need to obtain permission directly from the copyright holder. To view a copy of this license, visit http://creativecommons.org/ licenses/by/4.0/.
\end{abstract}

(C) The Author(s) 2020 